GRADIATION\&APPLICATIONS

ISSN 2466-4294 (online) | rad-journal.org

Vol. 3 | Issue 1 | pp. 27-33, 2018

doi: 10.21175/RadJ.2018.01.006

Original research paper

\title{
ASSESSMENT OF RADIATION EXPOSURE IN MANNED MISSIONS TO MARS FOR THREE PROFILES*
}

\author{
Ana Luísa Casimiro', ${ }^{1 * *}$, Jorge Miguel Sampaio',3, Patrícia Gonçalves ${ }^{1,2}$ \\ ${ }^{1}$ LIP - Laboratório de Instrumentação e Física Experimental de Partículas, Lisboa, Portugal \\ ${ }^{2}$ Instituto Superior Técnico, Universidade de Lisboa, Lisboa, Portugal \\ 3Faculadade de Ciências, Universidade de Lisboa, Lisboa, Portugal
}

\begin{abstract}
The biggest risks in a mission to Mars are the long periods of time with the lack of gravity, the psychological effects due to isolation, the risk of contamination by diseases in confined space, and exposure to high doses of radiation. It is recognized that the latter poses the greatest scientific and technological challenge to a viable mission. In this work, we present estimates of the equivalent dose in an astronaut for three mission-to-Mars profiles proposed by NASA. For this, we performed Monte Carlo simulations of the energy deposited in the ICRU sphere taking into account the main radiation sources in space using the Geant4 simulation toolkit. The results show that the introduction of $10 \mathrm{~cm}$ equivalent Al shielding significantly reduces the equivalent dose, although our estimates are still above the dose limits adopted by NASA. These results show, however, that the values are in the range for optimization in terms of shielding solutions, as well as the choice of the most appropriate mission trajectories to minimize the dose to astronauts.
\end{abstract}

Key words: Dose in astronauts, manned missions, Mars mission, radiation environment, simulations

\section{INTRODUCTION}

The human exploration of Mars is likely to be the biggest scientific and technological challenge in the coming decades. Getting astronauts to the Martian surface and returning them safely to Earth, however, is an extremely difficult engineering and safety challenge [1]. One of the biggest risks, if not the biggest risk to the health of astronauts, will be radiation exposure during the Earth's exit, cruise to Mars and stay on the planet's surface [2, 3].

The main radiation sources of concern are the trapped particles in the Van Allen (VA) belts, the galactic cosmic radiation (GCR), and the solar energetic particles (SEP) events. The GCR originates outside the Solar System and are an isotropic and continuous radiation source formed by nuclei accelerated to extremely high energies (up to $10^{20} \mathrm{eV}$ ). This radiation source is composed of about $98 \%$ baryons and $2 \%$ electrons, where the baryonic component is made of $\sim 85 \%$ protons ( $\mathrm{H}$ nuclei), $\sim 14 \%$ alpha particles ( $4 \mathrm{He}$ nuclei), and $\sim 1 \%$ of heavier nuclei [4]. The GCR fluxes are modulated by the interaction with the solar wind, which is coupled to the intensity of the magnetic field of the Sun. The 11-year (magnetic) solar activity cycles and the GCR fluxes are inversely correlated, that is, the GCR flux is maximum at the solar minimum and vice versa [5].
As a result of the interaction of the GCR and solar radiation with the Earth's magnetic field and atmosphere, charged particles become trapped in the magnetosphere, moving in spirals along the geomagnetic field lines until they are reflected back at the magnetic poles. The regions where the density of electrons and protons is much higher are known as the VA belts. The inner belt consists of electrons in the range of hundreds of $\mathrm{keV}$ and energetic protons with energies up to $1 \mathrm{GeV}$. These are formed in the decay of albedo neutrons produced by nuclear reactions of GCR and solar particles in the upper atmosphere and become trapped at an altitude between 200 and 6000 $\mathrm{km}$. The outer belt consists mainly of high energy (o.1$10 \mathrm{MeV}$ ) electrons trapped between 10 ooo and 75000 $\mathrm{km}$, although the size and composition of the outer belt can be strongly affected by geomagnetic storms related to solar flares and coronal mass ejections (CME) [6, 7].

Large SEP events are currently believed to be due to large, fast CMEs which can drive shocks in the low solar corona. These shocks are responsible for accelerating particles to relativistic energies $[8,9,10]$. These events are characterized by a sudden increase in the solar particle flux, up to several orders of magnitude higher than the GCR flux on time scales of tens of minutes to a few hours, and energies up to several hundred $\mathrm{MeV} /$ nucleon (in some cases up to a few $\mathrm{GeV} /$ nucleon). Their composition consists of

\footnotetext{
* The paper was presented at the Fifth International Conference on Radiation and Applications in Various Fields of Research (RAD 2017), Budva, Montenegro, 2017.

ana.luisa.casimiro@tecnico.ulisboa.pt
} 
intense electromagnetic radiation as well as charged particles, mainly protons, but also electrons and heavier ions (10\% helium and $1 \%$ of heavy ions), with energies ranging from a few tens of $\mathrm{keV}$ to $\mathrm{GeV}$. The probability of occurrence of a SEP is greatly reduced during the solar minimum, although they can still happen under these solar conditions. The stochastic nature of these events in terms of occurrence and flux magnitude makes them one of the most limiting factors in human space exploration.

Furthermore, Mars has a negligible magnetic field and the atmosphere has typical mass column densities of $20 \mathrm{~g} / \mathrm{cm}^{2}\left(\sim 22 \mathrm{~g} / \mathrm{cm}^{2}\right.$ at Gale Crater) which is about 1/50 of the Earth's column density of $1000 \mathrm{~g} / \mathrm{cm}^{2}$, putting the Mars surface above the Pfotzer maximum $[11,12]$. Thus, astronauts remaining a long time on the surface of the planet, without the adequate protection, will be exposed to primary GCR and possible SEP events, as well as to secondary particles produced in atmosphere and on the surface of the planet $[13,14]$. Crucial information on the Martian radiation environment is now available through measurements of the Radiation Assessment Detector (RAD) inside the Mars Science Laboratory (MSL), on board the Curiosity rover that landed in Gale Crater on August 6, 2012 [15].

This paper presents an estimate of radiation dose exposure of an astronaut for each of three preliminary mission scenarios proposed by the National Aeronautics and Space Administration (NASA) [16]. The Geant4 $[17,18]$ toolkit is used to simulate the energy deposited in the ICRU sphere [19], representing the astronaut, due to each of the radiation sources mentioned above. Doses from trapped particles are calculated with particle fluxes retrieved from SPENVIS [20] using a highly elliptical injection trajectory [21]. The same tool is used to obtain the GCR for solar minimum (January 30, 2009) and solar maximum (January 30, 2014) conditions used as primary fluxes in the calculation of the dose received during the cruise to Mars. To understand the contribution to the dose due to secondary particles produced in the spacecraft structure, simulations are done without and with shielding. For the latter, we consider a $10 \mathrm{~cm} \mathrm{Al} \mathrm{slab,}$ which corresponds to $\sim 27.8 \mathrm{~g} / \mathrm{cm}^{2}$ Al-equivalent, that is comparable to the average shielding of the RAD/MSL [22] during its 235-day cruise to Mars. Comparisons between measured and simulated shielded fluxes and doses in the relevant energy range and solar conditions during transit are used to benchmark our simulations. The Martian radiation environment is simulated with the dMEREM $[13,14]$ model, providing the particle spectra resulting from the interaction of GCR in the Martian atmosphere and soil at the coordinates of the Gale crater. These particle spectra are then used as the input fluxes in the simulations of the energy deposited in the sphere.

\section{Dose Calculations}

\subsection{Dose quantities}

Protection quantities [23] are based on mean absorbed doses to organs and tissues of the human body. These include the equivalent dose in a tissue or organ $\mathrm{T}$,

$$
H_{T}=\sum_{R} \omega_{R} D_{T, R},
$$

and effective dose,

$$
E=\sum_{T} \omega_{T} \frac{1}{2}\left(H_{T}^{M}+H_{T}^{F}\right),
$$

where $D_{T, R}$ is the mean absorbed dose from radiation type $\mathrm{R}$ in a tissue or organ $\mathrm{T}$, $\omega_{\mathrm{R}}$ is the radiation weighting factor that takes into account the relative biological effectiveness (RBE) of the radiation $\mathrm{R}$, and $\omega_{\mathrm{T}}$ is the tissue weighting factor that takes into account the relative contribution of the organ or tissue $\mathrm{T}$ to the total harm to the health (detriment). The equivalent dose limits are not established for an individual person but for Reference Computational Male $\left(\mathrm{H}_{\mathrm{T}}{ }^{\mathrm{M}}\right)$ and Female $\left(\mathrm{H}_{\mathrm{T}}^{\mathrm{F}}\right)$ [24]. However, the current recommendations [23] compute a single effective dose limit from the sex-averaged equivalent doses.

NASA adopts dose limits recommended in [25] that establish different effective dose limits for male and female astronauts as well as an age at exposure dependence (see Table 1). Equivalent dose limits for deterministic effects in astronauts are shown in Table 2 as a function of the time period of exposure.

Table 1. Effective dose limits for astronauts adopted by NASA

[25]. Stochastic effects limits correspond to a 3\% excess cancer mortality for 10-year careers.

\begin{tabular}{|c|c|c|}
\hline \multicolumn{3}{|c|}{ Stochasticeffects (effective dose limit in Sv) } \\
\hline Age at exposure (yr) & Male & Female \\
\hline 25 & 0.7 & 0.4 \\
35 & 1.0 & 0.6 \\
45 & 1.5 & 0.9 \\
55 & 3.0 & 1.7 \\
\hline
\end{tabular}

Table 2. Equivalent dose limits adopted by NASA [25]. Deterministic effects limits are frequently expressed in Grayequivalent (Gy-Eq) to account for RBE values that do not equal the stochastic limit values shown in Table 1.

Deterministic effects (equivalent dose in Gy-Eq)

\begin{tabular}{|c|c|c|c|}
\hline Time & BFO & Eye & Skin \\
\hline Career & (see Table 1) & 4.0 & 6.0 \\
1 year & o.6o & 2.0 & 3.0 \\
30 days & 0.25 & 1.0 & 1.5 \\
\hline
\end{tabular}

One important limitation of Eq. (1) is that the $\omega_{\mathrm{R}}$ depends only on the external field particle type and not on the characteristics of the internal field at the organ of interest. This limits the applicability of that equation in the case of neutron fields that are strongly modified by the transverse matter, or in the case of external mixed fields as it happens in space radiation exposures. Furthermore, for heavy ions with linear energy transfer (LET), L $>200 \mathrm{keV} / \mu \mathrm{m}$, the International Commission on Radiation Protection (ICRP) does not recommend the reference value of $\omega_{R}=20$ for ions. The preferred approach to compute the equivalent dose from exposure to high LET mixed space radiation is to use the LET-dependent equivalent dose $\mathrm{H}_{\mathrm{T}}(\mathrm{L})=\mathrm{Q}_{\mathrm{T}}(\mathrm{L}) \mathrm{D}_{\mathrm{T}}(\mathrm{L})$, 
A.L. Casimiro et al., Assessment of radiation exposure in manned missions to Mars..., Rad. Applic., 2018, 3, 1, 27-33

where $\mathrm{D}_{\mathrm{T}}(\mathrm{L})$ is the differential dose integrated in the volume of the organ or tissue $\mathrm{T}$, and $\mathrm{Q}(\mathrm{L})$ is the quality factor for the LET L. However, it is important to stress that the current lack of knowledge in the RBE for heavy ions, results in large uncertainties in predicting the value of $\mathrm{Q}(\mathrm{L})$ in space radiation environments.

In this work, we are not interested in accurate tissue or organ equivalent dose calculations, or in detailed evaluation of the effective dose in reference phantoms. The main goal is to compare dose estimates for different mission profiles and to assess the potential effect of secondary particles produced by shielding. For this, we assume a simple model, where the astronaut is modelled by the ICRU sphere immersed in an expanded and aligned external field. The corresponding equivalent dose is computed as,

$$
H_{I C R U}=\sum_{R} \omega_{R} D_{I C R U, R}=\sum_{R} \omega_{R}\left(\frac{\bar{\varepsilon}_{d e p}}{m}\right)_{I C R U, R}
$$

where $\mathrm{m}=14.137 \mathrm{~kg}$ is the mass of the ICRU sphere. The energy deposited flux inside the ICRU sphere is scored and integrated to give the total deposited energy, $\bar{\varepsilon}_{d e p}$, rate.

\subsection{Input radiation models}

The three mission profiles considered in this work differ in terms of exposure to radiation by the time spent in each of the mission stages (Earth exit and return entry, out- and inbound transit, and stay on Mars). The short-stay mission provides Mars stay times of 30 to 90 days with a round trip total time of 400 to 650 days. This mission requires a large amount of energy to be expanded in transit, even after taking advantage of either a Venus swing-by (on either the inbound or outbound leg) or a deep space propulsive maneuver in order to reduce Mars and Earth entry speeds. The Venus swing-by brings the spacecraft inside the orbit of Venus, where it would be subject to more intense solar particle flux and higher fluxes in case of a SEP event. Furthermore, the crew would spend $90 \%$ of the time in the zero-g space environment with little time for recovery in Mars.

Two long-stay missions are considered: i) a Mars stay up to 500 days with a round trip total time of about 900 days. The energy requirement for these missions is the lowest of the three mission profiles at the cost of a long transit time around 250 days. This trajectory provides an opportunity to send a larger payload but exposes the crew for a longer time to the space radiation environment; and ii) a Mars stay above 600 days with a total time for the round trip also of 900 days. This is only achievable with a significant increase in the propulsion energy in order to shorten transit time (one-way travel between 120 to 180 days). This fast transit mission maximizes the stay on Mars and reduces considerably the exposure time to space radiation. Table 3 summarizes the time spent in each stage of the three mission profiles as well as the most relevant radiation sources considered in the simulations.
Table 3. Time spent in each stage for three mission profiles proposed by NASA [16] and the radiation sources considered in the simulations

\begin{tabular}{|c|c|c|c|}
\hline $\begin{array}{c}\text { Mission } \\
\text { stage }\end{array}$ & $\begin{array}{c}\text { Earth } \\
\text { exit and } \\
\text { entry }\end{array}$ & $\begin{array}{c}\text { Out and } \\
\text { inbound } \\
\text { transit }\end{array}$ & Stay on Ma \\
\hline $\begin{array}{c}\text { Radiation } \\
\text { source }\end{array}$ & $\begin{array}{c}\text { VA } \\
\text { belts }\end{array}$ & GCR/SEP & $\begin{array}{c}\text { Mars radiation } \\
\text { environment } \\
\text { (from GCR/SEP) }\end{array}$ \\
\hline Short stay & $\begin{array}{c}1.5 \\
\text { hours }\end{array}$ & $224+291$ days & 30 days \\
\hline $\begin{array}{c}1.5 \\
\text { Long stay i) }\end{array}$ & $224+237$ days & 458 days \\
\hline $\begin{array}{c}\text { Long stay } \\
\text { ii) }\end{array}$ & $\begin{array}{c}1.5 \\
\text { hours }\end{array}$ & $150+110$ days & 619 days \\
\hline
\end{tabular}

The trapped particles and the GCR fluxes were retrieved from the SPENVIS system [20] that models the space environment along a trajectory or in a coordinate grid. For trapped particles, we used the AE8 (electrons) and AP-8 (protons) maps that contain omnidirectional integral fluxes in the energy range 0.04-7 MeV electrons and 0.1-400 MeV protons for solar maximum and solar minimum conditions. The GCR fluxes were obtained with the ISO-15390 model [26] that describes variations in $1-10^{5} \mathrm{MeV}$ GCR particles (electrons, protons, and $Z=2$ to 92 nuclei) in near-Earth space beyond the Earth's magnetosphere. This model accounts for variations in the fluxes with solar activity and in the large-scale heliosphere magnetic field (that are assumed to be proportional to the Sun's polar magnetic field) throughout 22-year cycles. The angular distribution beyond the Earth's magnetosphere is taken isotropic.

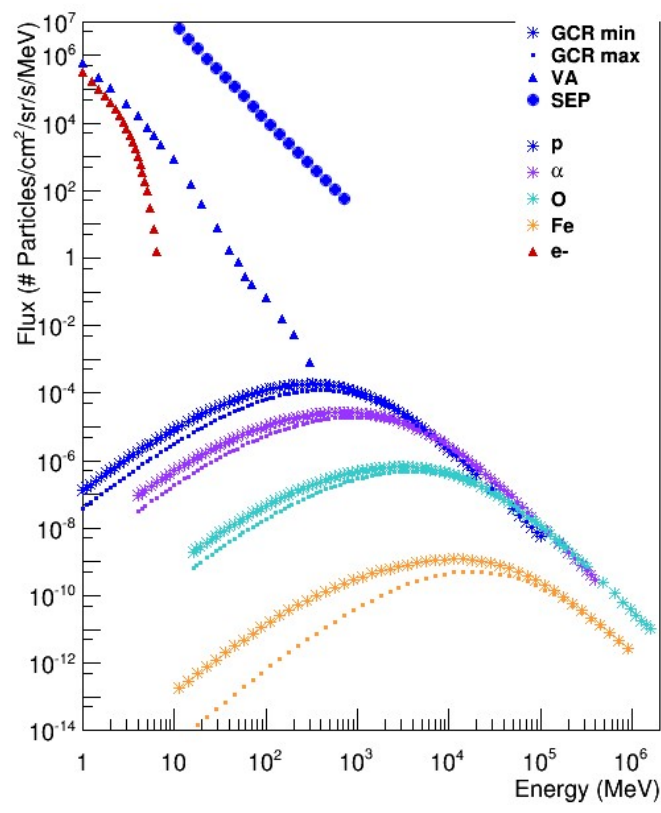

Figure 1. SPENVIS [20] primary particle fluxes used in this work for trapped particles (triangles) and for GCR (stars) at solar minimum (January 30, 2009) and solar maximum (January 30, 2014) conditions. The SEP (circle) event flux corresponds to the event observed in December 13, 2006. 
Fig. 1 shows the particle fluxes for the VA belts (electrons and protons) and the GCR (protons, alpha particles, oxygen and iron nuclei) used in our simulations. The GCR fluxes were evaluated at $1 \mathrm{AU}$ (taken as representative of the average GCR flux for a trajectory including a Venus swing-by) for the conditions of solar minimum (January 30, 2009) and solar maximum (January 30, 2014) of cycle 24. The SEP event proton flux used in our simulations, shown in the figure, corresponds to the measured flux of the intense, high-energy signal observed on December 13, 2006 [13].

The Martian radiation environment was obtained from ESA's model, dMEREM $[13,14]$ that calculates particle fluxes through detailed Monte Carlo simulations based on the Geant4 $[17,18]$ toolkit. Fluxes are simulated for primary and secondary particles in the energy range of the GCR fluxes, and for neutrons down to thermal energies. dMEREM includes a preprocessor to define the atmospheric density profile and composition, and another to specify the surface composition and density. For the present simulations, we used a $50 \mathrm{~km}$-high atmosphere composed of $\mathrm{CO}_{2}$ (corresponding 96\% of the total composition). The soil composition was obtained from in situ measurements by the Curiosity rover at the Gale crater [27, 28].

Fig. 2 shows the energy distribution of primary and secondary particle fluxes due to GCR protons predicted by dMEREM for the Gale Crater soil composition. The secondary particle fluxes are dominated by albedo neutrons down to the $\mathrm{eV}$ region and by a gamma contribution between a few tens of $\mathrm{keV}$ and $1 \mathrm{MeV}$. The high energy part of the spectrum is dominated by downward going protons and neutrons produced in the atmosphere.

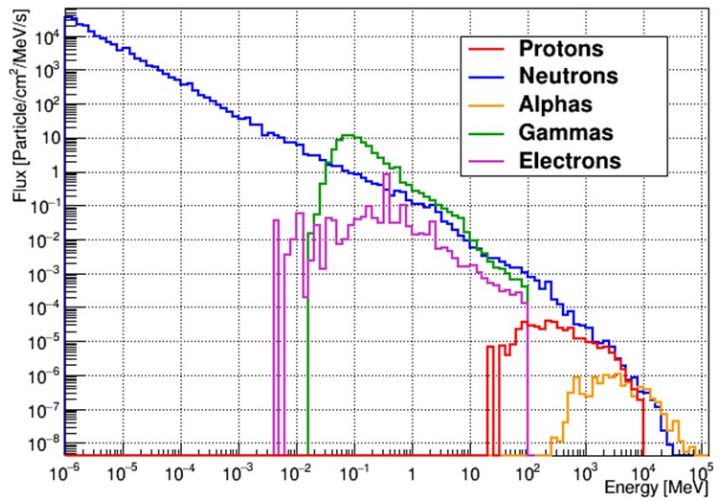

Figure 2. Primary and secondary particle fluxes obtained with dMEREM $[13,14]$ from GCR alpha particles near solar maximum (July 15, 2015) condition. These were used to assess the Martian radiation doses.

\subsection{Simulation parameters}

Simulations were carried out for expanded and aligned external fields of electrons in the energy range 0.1-50 $\mathrm{MeV}$ (VA belts); protons in the energy range 1-10 $3 \mathrm{MeV}$ (VA belts, SEP events, and Martian radiation environment); and protons, alpha particles, oxygen, and iron nuclei in the energy range $1-10^{5} \mathrm{MeV}$ (GCR). The latter two were taken as representative of the heavy ions composition in the GCR flux. Additional input fields (obtained from dMEREM simulations) of photons between $1 \mathrm{keV}$ and $90 \mathrm{MeV}$, and neutrons between $1 \mathrm{eV}$ and $1 \mathrm{TeV}$, were also considered in the simulations to assess the Martian radiation doses (see Fig. 2).

The energies of all particle types were sampled from a probability density function of the form $1 / \varepsilon$, where $\varepsilon$ is the energy of the primary particle. The simulated deposited energy flux was than weighted with a factor that normalizes this probability density function to the flux of the radiation source under consideration. The number of simulated primary particles was $10^{6}$. The number of particles that deposited energy in the sphere contributing to the dose was used to determine the statistical uncertainty in our calculations. The average uncertainty in dose values was estimated to be $\sim 0.5 \%$.

Due to the low energy albedo neutrons present on Mars, a Geant4 physics model that contains a high precision neutron package to transport neutrons below $20 \mathrm{MeV}$ down to thermal energies was used in all simulations (Geant 4 Physics list QGSP_BERT_HP).

\section{RESULTS AND DISCUSSION}

\subsection{Validation with RAD/MSL data during cruise to Mars}

Charged particle spectra were measured during the MSL cruise to Mars with the RAD [29] mounted on board the Curiosity rover inside the spacecraft. The rover was placed between the heat shield (below the rover) and the descent stage (above the rover). The percentage of shielding for different $\mathrm{Al}$ equivalent area densities in the RAD field-of-view (FOV) are reported in [22]. The values range between 1 and $85 \mathrm{~g} / \mathrm{cm}^{2}$ with a (weighted) mean of $\sim 27.5 \mathrm{~g} / \mathrm{cm}^{2}$ and median of $\sim 5$ $\mathrm{g} / \mathrm{cm}^{2}$. However, one should note that the different components in the MSL around the RAD provide a very heterogeneous shielding distribution in the FOV (see supplementary material in [30]).

Figure 3 shows the comparisons between the simulation of stopping protons flux from GCR and the measured flux by RAD between June 11 and July 14, 2012. The simulations were done with the Geant4 based software package MULASSIS from SPENVIS [20]. The free space proton and alpha particles GCR fluxes were also taken from SPENVIS for June 30, 2012 (variations of GCR fluxes in the measurement period are small). Simulations were done for $5 \mathrm{~g} / \mathrm{cm}^{2}$ (RAD median shielding value), $10 \mathrm{~g} / \mathrm{cm}^{2}$ (Al-equivalent shielding depth that better reproduces RAD measurements) and $27.5 \mathrm{~g} / \mathrm{cm}^{2}$ (RAD weighted average shielding) $\mathrm{Al}$ thickness. The $10 \mathrm{~g} / \mathrm{cm}^{2} \mathrm{Al}$-equivalent shielding depth corresponds to a division in the shielding distribution of $52.5 \%-47.5 \%$ (very similar to median value $50 \%-50 \%$ ). The highest flux is obtained for a shielding depth corresponding to $27.5 \mathrm{~g} / \mathrm{cm}^{2}$ whereas the lowest flux is for $5 \mathrm{~g} / \mathrm{cm}^{2}$. The secondary proton flux is produced by nuclear proton and alpha reactions on Al. For thin shielding $\left(5 \mathrm{~g} / \mathrm{cm}^{2}\right.$ in this case), the alpha reactions do not contribute as much as for thick shielding $\left(27.5 \mathrm{~g} / \mathrm{cm}^{2}\right)$ to the secondary protons. In the energy range considered (10-100 MeV) 
the proton stopping power is dominated by the electronic component and their range varies between 0.17 and $10 \mathrm{~g} / \mathrm{cm}^{2}$. Thus, for $\mathrm{Al}$ equivalent shielding above $\sim 10 \mathrm{~g} / \mathrm{cm}^{2}$, the high energy component of the secondary proton flux decreases considerably as more protons are down-scattered to the lower component.

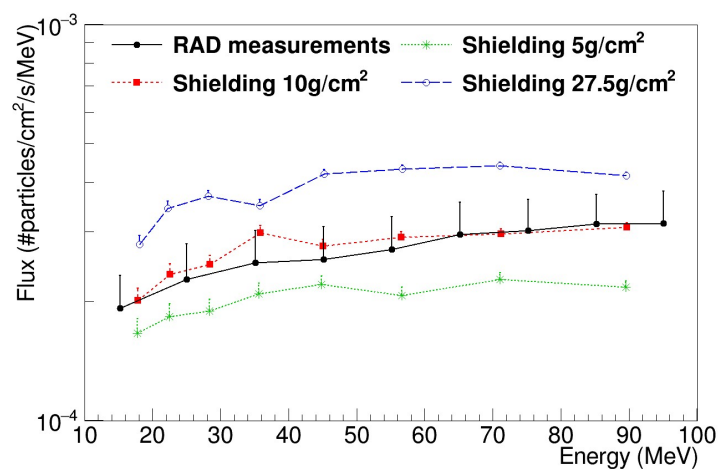

Figure 3. Comparison between the stopping protons flux measured by RAD and the mean, median and optimal shielded proton fluxes from Al simulated with MULASSIS and Geant4.

\subsection{Equivalent dose for each mission profile}

Equivalent doses for each mission profile were determined from the absorbed dose rates for each particle type and integrating it over the time periods of each mission stage as shown in Table 3. The total equivalent doses were then estimated with Eq. (2) for solar minimum and solar maximum conditions. The results are shown in Fig. 4 for simulations without shielding and in Fig. 5 for simulations with a $10 \mathrm{~cm} \mathrm{Al}$ slab to simulate the spacecraft structure.

Without shielding the total mission, the equivalent dose limits are exceeded in all three scenarios by more than one order of magnitude, whereas with shielding the total equivalent dose decreases on average $67 \%$. Still, the total equivalent dose exceeds, on average, the maximum dose limit in about $77 \%$. It should be noted that the contribution of the VA belts decreases almost 11300 times when shielding is considered, since up to $100 \mathrm{MeV}$ these particles are stopped by the $\mathrm{Al}$ slab. This makes the GCR the most significant contributor to the mission equivalent dose when shielding is considered, with about $92 \%$ on average over the three mission profiles.

Furthermore, no significant differences were found between the GCR contributions for solar minimum and for solar maximum conditions. We note that our calculations were done for solar maximum and solar minimum conditions in cycle 24 , corresponding to solar modulation potentials of $\Phi=606 \mathrm{MV}$ (January 2014) and $\Phi=302$ MV (January 2009), respectively. For these potentials, there are small differences in the flux for solar maximum and solar minimum conditions below $1 \mathrm{GeV}$, as shown in Figure 1, and hence also no large differences are found in the dose $(\sim 25 \%$ difference). Guo et al. [31] computed the doses for solar maximum with $\Phi=1200 \mathrm{MV}$ in cycle 22 and solar minimum with $\Phi=250 \mathrm{MV}$ in cycle 24 . Therefore these authors had got a much larger difference than the one obtained in our calculations. Using the linear correlation of the dose with the solar modulation potential, as proposed by these authors, $\mathrm{D}=755^{-0.44} \Phi$, where the fit constants correspond to the response for the stopping particles in $\mathrm{RAD}$, the difference is just $\sim 27 \%$ for the solar conditions used in our simulations. The unshielded dose rate for near-Earth interplanetary space in the solar minimum of 2009 was estimated by Mrigakshi et al. [32] to be $370 \mu \mathrm{Gy} /$ day, using the BON2010 model, $395 \mu \mathrm{Gy} /$ day with the CREME 96 model, $398 \mu \mathrm{Gy} /$ day, using CREME 2009 model, $469 \mu \mathrm{Gy} /$ day with the Mathiô/Oulu model, and $472 \mu \mathrm{Gy} /$ day using the Mathiò/ACE GCR model. These values are close to the value of $564 \mu \mathrm{Gy}$ /day that we estimated using the ISO-15390 model. Mrigakshi et al. [32] obtained a factor of 3-4.5 between dose rates in near-Earth interplanetary space, however, this resulted from comparing the 2009 solar minimum and the solar maxima from January 1970 to October 2011.
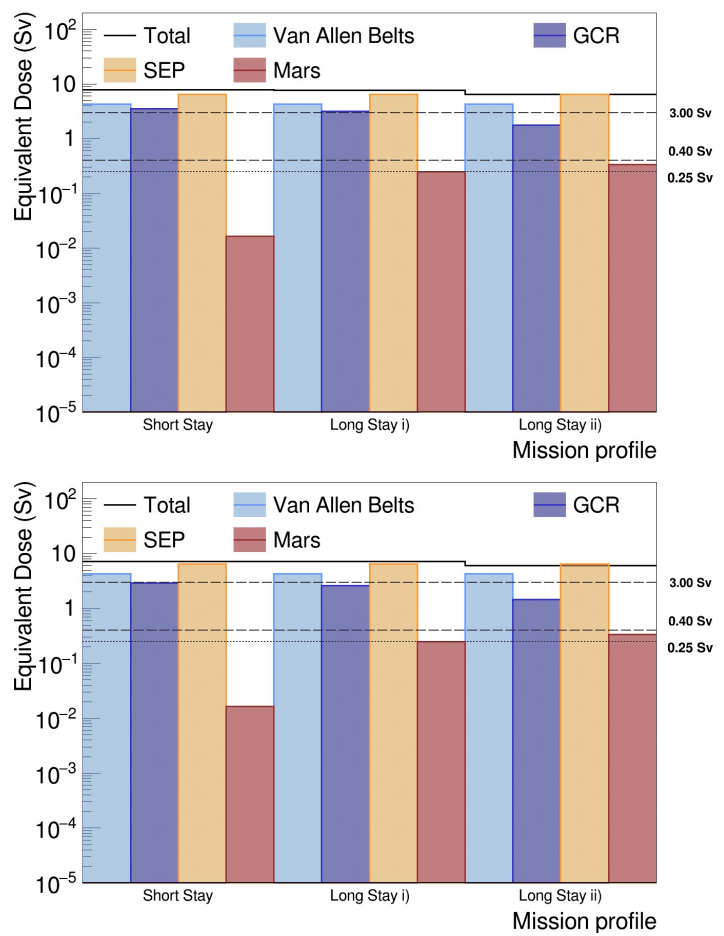

Figure 4. Equivalent dose for each mission profile and radiation environment in a) minimum and b) maximum solar conditions without shielding (full black lines represent the total equivalent dose and the dashed lines represent the dose limits range for astronauts from Table 1).

The proton SEP flux observed in December 13, 2006 (see Fig. 1) was also simulated to estimate a 14 days integrated dose if one of these events occurs during transit. The unshielded dose from the SEP event is $6.40 \mathrm{~Sv}$. Since these events develop in a very short period of time, this value must be compared against the deterministic dose limits for 30-day exposure shown in Table 2. Thus, without shielding, the SEP equivalent dose exceeds both eye and skin limits by a large factor. However, when considering shielding, the equivalent dose from the proton SEP reduces drastically to less 
than $20 \mathrm{mSv}$, due to the short range of protons below $1 \mathrm{GeV}$ in $\mathrm{Al}$.
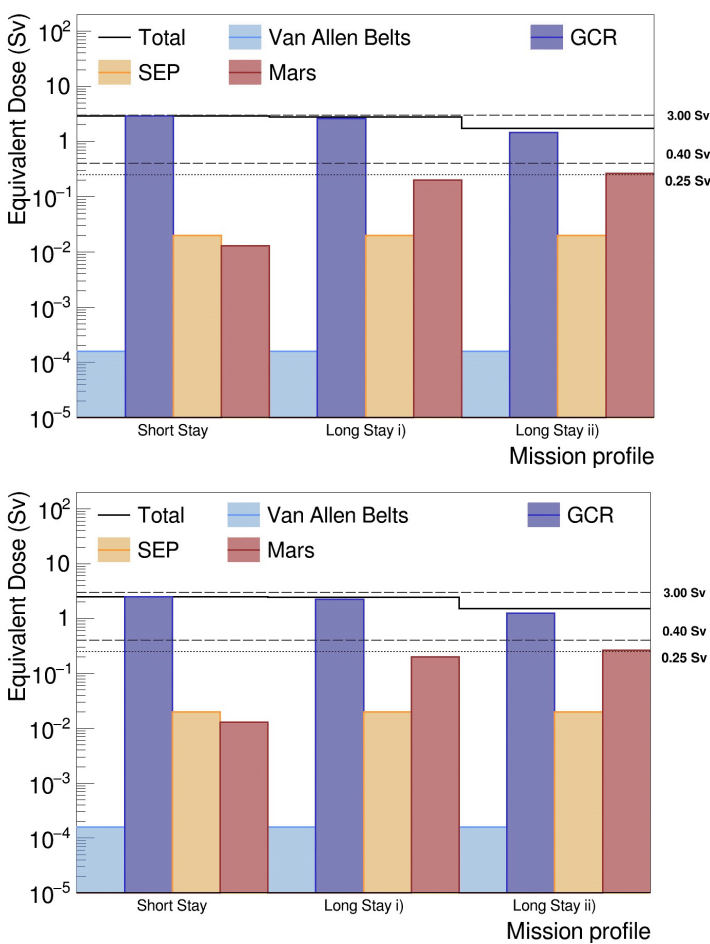

Figure 5. Equivalent dose for each mission profile and radiation environment in a) minimum and b) maximum solar conditions with a $10 \mathrm{~cm} \mathrm{Al}$ shielding (full black lines represent the total equivalent dose and the dashed lines represent the dose limits range for astronauts from Table 1).

\section{CONCLUSIONS}

In this work, the radiation dose exposure of an astronaut in terms of equivalent dose in the ICRU sphere were estimated for three mission to Mars profiles proposed by NASA. For this, the Geant4 toolkit was used together with the relevant input particle fluxes retrieved from the SPENVIS and a measured particle flux for the SEP event. The simulations were validated against measurements with RAD/MSL data during cruise to Mars.

Table 4. Summary of the equivalent doses calculated in this work (Min. and Max. designate minimum and maximum solar conditions, respectively).

\begin{tabular}{|l|c|c|c|c|}
\hline \multirow{2}{*}{} & \multicolumn{3}{|c|}{ Equivalent dose (Sv) } \\
\cline { 2 - 5 } & No shielding & \multicolumn{2}{c|}{ With shielding } \\
\hline Profiles & Min. & Max. & Min. & Max. \\
\hline Short stay & 7.83 & 7.22 & 2.93 & 2.51 \\
Long stay i) & 7.69 & 7.14 & 2.81 & 2.44 \\
Long stay ii) & 6.40 & 6.09 & 1.74 & 1.53 \\
\hline SEP event & \multicolumn{2}{|c|}{6.40} & \multicolumn{2}{c|}{0.02} \\
\hline
\end{tabular}

Table 4 summarizes our results. It is clear that adequate shielding is mandatory to make a mission to Mars viable. Although equivalent dose values with a 10 $\mathrm{cm} \mathrm{Al}$ shielding are still above NASA limits, they are in a range where it is foreseeable that optimization in passive shielding materials composition layouts, as well as active shielding (using superconducting magnetic coils to deflect charged particles), and optimized trajectories can lower them to the acceptable range.

The contribution from the December 2006 SEP (20 mSv) with shielding is under the 30-day limit for deterministic effects for the BFO, eyes lens and skin. However, due to the stochastic nature of these events in terms of the number occurrences and flux magnitude, doses from SEP events put the most serious exposure risks on a long space mission. In addition, many activities on the surface of Mars will be made outside the shelters, without adequate protection in the event of a SEP event. It is, therefore, crucial to develop reliable SEP forecast models.

For the conditions of the solar cycle 24 used in this work, there is no significant difference between predicted doses due to GCR in solar minimum and solar maximum conditions, because the solar maximum of January 2014 is atypically weaker than in the previous cycles. However, we notice that the higher probability of SEP occurrence during solar maxima must also be considered in the choice of mission dates.

Future assessments of radiation exposure in manned missions to Mars should include organ equivalent dose calculations using a detailed anthropomorphic computational phantom in the simulations. Other materials and more complex shielding structures should also be considered. These simulations are useful to optimize mission profiles in order to reduced radiation exposure. However, one should point out that even for very detailed and realistic simulations, they will result in equivalent doses estimates with a high degree of uncertainty, since the quality factors for heavy ions are poorly known. This calls for a greater investment in studies of radiobiology with high LET ions to improve the knowledge of their RBE.

\section{REFERENCES}

1. NASA's Journey to Mars: Pioneering Next Steps in Space Exploration, NASA, Washington (DC), USA 2015.

Retrieved from:

https://www.nasa.gov/sites/default/files/atoms/files/j ourney-to-mars-next-steps-20151008 508.pdf;

Retrieved on: Dec. 10, 2017

2. S. M.-Lawlor et al., "Overview of energetic particle hazards during prospective manned missions to Mars," Planet. Space Sci., vol. 63-64, pp. 123 - 132, Apr. 2012. DOI: 10.1016/j.pss.2011.06.017

3. M. Durante, "Space radiation protection: Destination Mars," Life Sci. Space Res., no. 1, pp. 2 - 9, Apr. 2014. DOI: 10.1016/j.lssr.2014.01.002 PMid: 26432587

4. J. A. Simpson, "Elemental and isotopic composition of the galactic cosmic rays," Ann. Rev. Nucl. Part. Sci. vol. 33, pp. $323-381$, Dec. 1983.

DOI: 10.1146/annurev.ns.33.120183.001543

5. Assessment of radiation exposure of astronauts in space, ICRP Publication 123, ICRP, Ottawa, Canada, 2013.

DOI: 10.1016/j.icrp.2013.05.004

PMid: 23958389 
A.L. Casimiro et al., Assessment of radiation exposure in manned missions to Mars..., Rad. Applic., 2018, 3, 1, 27-33

6. N. Yu Ganushkina et al., "Locations of boundaries of outer and inner radiation belts as observed by cluster and double star," J. Geophys. Res. A, vol. 116, no. A9, A09234, Sep. 2011. DOI: $10.1029 / 2010 J A 016376$

7. D. V. Reames, "The two sources of solar energetic particles," Space Sci. Rev., no. 175, no. 1-4, pp. 53 - 92, Jun. 2013. DOI: $10.1007 / \mathrm{s} 11214-013-9958-9$

8. S. K. Antiochos et al., "A model for solar coronal mass ejection," Astrophys. J., vol. 510, no. 1, pp. $485-493$, Jan. 1999. DOI: $10.1086 / 306563$

9. M. J. Aschwanden, "The localization of particle acceleration sites in solar flares and CMEs," Space Sci. Rev., vol. 124, no. 1-4, pp. 361 - 372, Jun. 2006. DOI: $10.1007 / \mathrm{s} 11214-006-9095-9$

10. N. Gopalswamy et al., "Type II radio bursts and energetic solar eruptions," J. Geophys. Res.-Space, vol. 110, no. A9, Ao9soo, Sep. 2005. DOI: 10.1029/2005JA011158

11. S. C. R. Rafkin et al., "Diurnal variations of energetic particle radiation at the surface of Mars as observed by the Mars Science Laboratory Radiation Assessment Detector," J. Geophys. Res.-Planet, vol. 119, no. 6, pp. $1345-1358$, Jun. 2014. DOI: $10.1002 / 2013 J E 004525$

12. Wimmer-Schweingruber et al., "On Determining the Zenith-Angle Dependence of the Martian Radiation Environment at Gale Crater Altitudes," Geophys. Res. Lett., vol. 42, no. 24, pp. 10557 - 10564, Dec. 2015. DOI: 10.1002/2015GL066664

13. S. M.-Lawlor et al, "Characterization of the particle radiation environment at three potential landing sites on Mars using ESA's MEREM models," Icarus, vol. 218, no. 1, pp. 723 - 734, Mar. 2012. DOI: $10.1016 /$ j.icarus.2011.04.004

14. P. Gonçalves et al., "MARSREM: the Mars Energetic Radiation Environment Models," in Proc. 31st Int. Cosmic Ray Conf. (ICRC), Łódź, Poland, 2010, pp. 1-4.

15. D. M. Hassler et al., "Mars' surface radiation environment measured with the Mars Science Laboratory's curiosity rover," Science, vol. 343, no. 6169, 1244797, Jan. 2014.

DOI: $10.1126 /$ science.1244797 PMid: 24324275

16. D. R. Williams, A crewed mission to Mars, NASA, Washington (DC), USA, 2015. Retrieved from: http://nssdc.gsfc.nasa.gov/planetary/ mars/marsprof.html; Retrieved on: Oct. 10, 2016

17. S. Agostinelli et al., "Geant4- simulation toolkit," Nucl. Instr. Meth. Phys. Res. A, vol. 506, no. 3, pp. $250-$ 303, Jul. 2003.

DOI: 10.1016/So168-9002(03)01368-8

18. J. Allison et al., "Geant4 developments and applications," IEEE Trans. Nucl. Sci., no. 53, no. 1, pp. $270-278$, Feb. 2006. DOI: 10.1109/TNS.2006.869826

19. Radiation Quantities and Units, ICRU Rep. 33, ICRU, Washington (DC), USA, 1980. DOI: $10.1002 /$ jlcr.2580180918

20. S. Calders et al., Space environment information system (SPENVIS) version 4.6.9, European Space Agency, Paris, France, 2017.

Retrieved from: https://www.spenvis.oma.be;

Retrieved on: Jan. 17, 2018
21. R. A. Braeunig, "Apollo 11's translunar trajectory and how they avoided the heart of the radiation belts," braeuing.us, Mar. 24, 2016.

Retrieved from: http://www.braeunig.us/apollo/apollo 11TLI.htm;

Retrieved on: Oct. 10, 2016.

22. B. Ehresmann et al., "Charged particle spectra measured during the transit to Mars with the Mars Science Laboratory Radiation Assessment Detector (MSL/RAD)", Life Sci. Space Res., no. 10, pp. 29 - 37, Aug. 2016.

DOI: 10.1016/j.lssr.2016.07.001

PMid: 27662785

23. The 2007 Recommendations of the International Commission on Radiological Protection, ICRP Publication 103, ICRP, Ottawa, Canada, 2007.

Retrieved from: https://www.sciencedirect.com/journal /annals-of-the-icrp/vol/37/issue/2;

Retrieved on: Oct. 10, 2016

24. Basic Anatomical and Physiological Data for Use in Radiological Protection Reference Values, ICRP Publication 89, ICRP, Ottawa, Canada, 2002. Retrieved from: https://www.sciencedirect.com/journal /annals-of-the-icrp/vol/32/issue/3;

Retrieved on: Oct. 10, 2016

25. Radiation Protection Guidance for Activities in LowEarth Orbit, Report No. 132, NCRP, Bathesda (MD), USA, 2000.

26. Space environment (natural and artificial) - Galactic cosmic ray model, ISO 15390, May, 2004. Retrieved from: http://www.spacewx.com/Docs/ISO P RF 15390 E.PDF;

Retrieved on: Oct. 10, 2016

27. S. M. McLennan et al, "Elemental geochemistry of sedimentary rocks at Yellowknife Bay, Gale Crater, Mars," Science, vol. 343, no. 6169, 1244734, Jan. 2014. DOI: $10.1126 /$ science.1244734

PMid: 24324274

28. D. M. Hassler et al, "Mars' surface radiation environment measured with the Mars Science Laboratory's Curiosity rover," Science, vol. 343, no. 6169, 1244797, Jan. 2014.

DOI: $10.1126 /$ science.1244797 PMid: 24324275

29. D. M. Hassler et al., "The Radiation Assessment Detector (RAD) investigation," Space Sci. Rev., vol. 170, no. 1-4, pp. 503 - 558, Sep. 2012 DOI: $10.1007 / \mathrm{s} 11214-012-9913-1$

3o. C. Zeitlin et al., "Measurements of energetic particle radiation in transit to Mars on the Mars Science Laboratory," Science, vol. 340, no. 6136, pp. $1080-$ 1084, May 2013.

DOI: $10.1126 /$ science. 1235989 PMid: 23723233

31. J. Guo et al., "Variations of dose rate observed by MSL/RAD in transit to Mars," Astron. Astrophys., vol. 577, A58, May 2015. DOI: 10.1051/0004-6361/201525680

32. A. Mrigakshi et al., "Estimation of Galactic Cosmic Ray exposure inside and outside the Earth's magnetosphere during the recent solar minimum between solar cycles 23 and 24," Adv. Space Res., vol. 52, no. 5, pp. $979-$ 987, Sep. 2013. DOI: 10.1016/j.asr.2013.05.007 